\title{
Spatio-temporal Brain Dynamics Underlying Saccade Execution, Suppression, and Error-related Feedback
}

\author{
Anthony T. Herdman ${ }^{1,2}$ and Jennifer D. Ryan ${ }^{1}$
}

\begin{abstract}
- Human and nonhuman animal research has outlined the neural regions that support saccadic eye movements. The aim of the current work was to outline the sequence by which distinct neural regions come on-line to support goal-directed saccade execution and error-related feedback. To achieve this, we obtained behavioral responses via eye movement recordings and neural responses via magnetoencephalography (MEG), concurrently, while participants performed an antisaccade task. Neural responses were examined with respect to the onset of the saccadic eye movements. Frontal eye field and visual cortex activity distinguished subsequently successful goal-directed saccades from (correct and erroneous) reflexive saccades prior to
\end{abstract}

\section{INTRODUCTION}

Looking toward a target requires coordination between systems that process target location information and those that govern eye movement control (Mays \& Sparks, 1980; Schiller, Stryker, Cynader, \& Berman, 1974). Singleunit recordings from primates have outlined the temporal dynamics of neural activation in relation to the execution and correction of eye movement behavior (Lee, Rohrer, \& Sparks, 1988; Hikosaka \& Wurtz, 1985; Schiller, True, \& Conway, 1980; Schiller \& Stryker, 1972). Activation in the saccade neurons of the primate superior colliculus and frontal eye fields (FEFs) occurs prior to saccadic onset (Everling \& Fischer, 1998). Activation within the fixation neurons of the superior colliculus and FEF precedes successful inhibition of reflexive eye movement behavior; errors in inhibiting a reflexive response are related to the imbalance of firing between saccade and fixation neurons (Munoz \& Everling, 2004; Everling \& Munoz, 2000). Following an erroneous reflexive saccade, FEF activity is sustained, indicating the possible generation of an errorrelated feedback signal that drives a corrective saccade to the task-relevant goal location (Bruce \& Goldberg, 1985).

\footnotetext{
${ }^{1}$ Rotman Research Institute, Baycrest, Toronto, ${ }^{2}$ Thompson Rivers University, British Columbia, Canada
}

the deployment of the eye movement. Activity in the same neural regions following the saccadic movement distinguished correct from incorrect saccadic responses. Error-related activity in the frontal eye fields preceded that from visual regions, suggesting a potential feedback network that may drive corrective eye movements. This work provides the first empirical demonstration of simultaneous remote eyetracking and MEG recording. The coupling of behavioral and neuroimaging technologies, used here to characterize dynamic brain networks underlying saccade execution and error-related feedback, demonstrates a novel within-paradigm converging evidence approach by which to outline the neural underpinnings of cognition.

Corresponding human neuropsychological research has also documented roles for the superior colliculus and FEF in mediating reflexive and goal-directed eye movements, respectively (Pierrot-Deseilligny, Rivaud, Gaymard, \& Agid, 1991; Guitton, Buchtel, \& Douglas, 1985). As well, the dorsolateral prefrontal cortex (DLPFC) has been shown to be critical for the successful suppression of reflexive eye movements. Patients who have lesions to the DLPFC, children with underdeveloped frontal lobes, and older adults who likely have atrophy within the frontal lobes, all show impaired performance on the antisaccade task in which goal-generated saccades must be executed to the opposite location from an abrupt target onset (Munoz, Broughton, Goldring, \& Armstrong, 1998; PierrotDeseilligny et al., 1991; Guitton et al., 1985).

Neuroimaging studies in neurologically intact humans using positron emission tomography (PET) or functional magnetic resonance imaging (fMRI) provide converging evidence regarding the neural regions underlying saccadic control. Activations in prefrontal regions have been found to be significantly greater for goal-directed versus reflexive saccades (Ford, Goltz, Brown, \& Everling, 2005; Cornelissen et al., 2002; Sweeney et al., 1996). In particular, Connolly, Goodale, Menon, and Munoz (2002) revealed larger blood-oxygenation-level-dependent signals within the FEF for goal-directed versus reflexive saccades, 
suggesting a role for this region in the intention and preparation for an eye movement response.

Larger neural activation for goal-directed versus reflexive saccades has also been observed in posterior brain regions, particularly within the cuneus, which has been thought to be responsible for the maintenance of the spatial information corresponding to the eye movement target location (Matsuda et al., 2004; Kimmig, Greenlee, Huethe, \& Mergner, 1999; Dorrichi et al., 1997). A convincing demonstration of the role of the parieto-occipital cortex in the coding of eye position comes from Law, Svarer, Rostrup, and Paulson (1998), in which activation was observed in the cuneus of subjects who were required to generate eye movements in the dark, and therefore, in the absence of any visual stimulation.

Although it is clear from the above work that activation within frontal and posterior regions supports reflexive and goal-directed eye movements, the relative temporal sequence by which these neural regions come on-line prior to saccade onset to support such eye movement behavior in humans remains largely unknown (Munoz \& Everling, 2004). Although neural activity can be roughly classified to time periods that are either presaccade or postsaccade execution, PET and fMRI techniques do not have adequate temporal resolution to outline the time course by which distinct neural regions come on-line with respect to saccade onset. Moreover, observed presaccadic and postsaccadic neural responses that differentiate reflexive from goal-directed eye movements could be confounded due to differences in saccade latency, as research has shown that antisaccades take longer to initiate compared with prosaccades (Olk \& Kingstone, 2003). If measurements of neural activity are not time-locked with saccade onset, saccade-specific responses may be temporally smoothed using techniques such as fMRI and PET.

Electroencephalographic (EEG) recordings have superior temporal resolution to PET and fMRI, making it a useful technique to examine the timing of neural responses with respect to saccade onset. However, the spatial localization is less precise because the electrical signals are obscured by skull and tissue. For instance, Everling, Krappmann, and Fiohr (1997) found neural responses originating from frontal electrodes that distinguished prosaccades from antisaccades prior to the onset of the saccade; based on converging evidence from human neuropsychological and primate work, it is assumed that these signals likely originate from within the FEF and/or DLPFC (Clementz, McDowell, \& Stewart, 2001; Kurtzberg \& Vaughan, 1982).

By contrast, MEG has the same temporal resolution as EEG, but magnetic fields are not susceptible to attenuation by skull and tissue, therefore, its spatial localization is more precise than EEG. A recent study by McDowell et al. (2005) used EEG and MEG to conjointly characterize the neural regions that support reflexive and goal-directed eye movements. In that study, subjects performed prosaccade and antisaccade trials in separate blocks in which a central fixation cross was presented for $2000 \mathrm{msec}$, followed by the onset of a target stimulus for either $500 \mathrm{msec}$ (prosaccade trials) or $1000 \mathrm{msec}$ (antisaccade trials), and then a blank screen for 500 msec. Analyses that were locked to the onset of the saccades revealed neural activity originating from regions such as the FEF, DLPFC, and the cuneus that was greater for antisaccade trials compared to prosaccade trials prior to saccadic onset. However, the timing by which these regions came on-line with respect to one another to support saccadic behavior was not discussed. Although the McDowell et al. study revealed important spatio-temporal differences between antisaccades and prosaccades, the neural activity to erroneous responses was not analyzed. Therefore, questions remain regarding the spatiotemporal dynamics of error-related feedback in addition to the relative sequence of presaccadic neural activation.

The goals of the present work were to describe the relative timing of neural activity in humans that governs and suppresses reflexive eye movement behavior and to particularly outline the progression by which neural regions come on-line to generate error-related feedback signals. To achieve this, we obtained recordings simultaneously from eye movement monitoring (EMM) and MEG while participants performed an antisaccade task in which they were required to make a reflexive eye movement toward an abrupt onset (prosaccade), or make a goal-directed eye movement away from an abrupt onset (antisaccade). Although it was expected that similar neural regions would be recruited for saccade execution and suppression in the current work, as observed in the aforementioned studies, no a priori predictions were made regarding temporal sequence of neural activity.

Eye movement monitoring provided an index of behavior regarding the extent to which goal-directed saccades were produced and reflexive saccades were inhibited. Although saccades can be clearly distinguished in the MEG field data, EMM data provide complimentary information of saccade direction, velocity, and distance that is absent in the MEG data. EMM data were used to classify MEG trials as those in which correct and erroneous, prosaccades and antisaccades, were generated. The timing information provided by EMM regarding when a saccade occurred was used to isolate the same time interval in the MEG data stream. Analysis of MEG data prior to this time interval provided information regarding the brain regions that support the signals to move the eyes (either in a reflexive or goaldirected manner) or to inhibit a reflexive eye movement on antisaccade trials. Analysis of MEG following saccadic onset for correctly executed versus erroneous saccades provided information regarding the neural responses underlying saccade execution and error-related feedback. EMM provided behavioral descriptions of cognitive phenomena such as successful and unsuccessful inhibition of the reflexive eye movement, whereas 
MEG provided temporal information regarding dynamic brain networks underlying such processing. The coupling of behavioral and neuroimaging technologies, used here to characterize dynamic brain networks underlying saccade execution and error-related feedback, demonstrates a novel within-paradigm converging evidence approach by which information is obtained concurrently from multiple methodologies to describe cognition and the neural underpinnings of behavior.

\section{METHODS}

\section{Participants}

Eleven young adults (age 18-34 years; average age 24.0 years) with normal neurological status participated in exchange for monetary compensation. Ten of the participants were right-hand dominant and one was lefthand dominant as assessed by the Edinborough handedness test. Data from the left-handed participant were not markedly different from right-handed participants and were therefore included in further analyses.

\section{Procedure}

Participants were required to randomly perform 200 prosaccades and 200 antisaccades. The following trial procedure is similar to that from our previous work (Bialystok, Craik, \& Ryan, 2006). A crosshair appeared in the center of the screen and remained for $400 \mathrm{msec}$. A caricatured face that served as the saccade cue was then presented, flanked by a box on either side. The eyes within the face were either green or red, which cued the participants to make either a prosaccade or an antisaccade, respectively, to a briefly flashed target. The two boxes (edged lengths of $3^{\circ}$ visual angle) on either side of the face were centered at $10^{\circ}$ of visual angle to the left and to the right of the center fixation. The face cue and the boxes were presented for $1000 \mathrm{msec}$, after which the boxes alone remained on the screen for an additional $200 \mathrm{msec}$. The removal of the central face cue $200 \mathrm{msec}$ prior to the presentation of the target served as a gap condition, so as to disengage central fixation and increase variability in saccade performance (Forbes \& Klein, 1996; Dorris \& Munoz, 1995). An asterisk target was then randomly presented in the center of either the left or right box at equal probabilities across the experiment. The asterisk remained on the screen for $200 \mathrm{msec}$, after which empty boxes remained on the screen for an average duration of $1500 \mathrm{msec}$ (a randomly distributed duration of 1250 to $1750 \mathrm{msec}$ ). Upon the appearance of the asterisk, participants were required to make a saccade toward the box where the asterisk appeared if the face cue's eyes were green (i.e., prosaccade), or to the box opposite of where the asterisk appeared if the eyes were red (i.e., antisaccade). The asterisk was displayed for only $200 \mathrm{msec}$ so that there would be no difference in the visual input during the saccade movement and/or when the eyes fixated on the target location in the prosaccade and antisaccade trials. Such differences in visual input could otherwise create differences in any observed postsaccadic responses. The offset interval was randomized so that the appearance of the fixation crosshair would be less predictable, and therefore, the onset of the crosshair would be more likely to elicit an orienting saccade toward the center of the display in preparation for the subsequent trial.

\section{EMM-MEG Measurements and Analyses}

We simultaneously recorded eye movements and magnetic fields using EMM and MEG in a magnetically shielded room while participants performed the antisaccade task. Participants were seated comfortably in an upright position in the MEG unit with their back supported. Eye movement and video monitoring verified alertness and task compliance.

\section{Eye Movement Monitoring}

Eye movements were recorded using an Applied Sciences Laboratory (ASL-504) remote infrared, camerabased eyetracker. The eyetracker recorded changes in the angle of light reflected from the pupil and corneal reflection to determine eye position. A 9-point calibration procedure was conducted prior to the experiment and the initiation of neuromagnetic recordings. Following calibration, in order to reduce electromagnetic noise from the eyetracker that would contaminate MEG recordings, automatic focus and tracking settings were disabled. Given participant restraint within the MEG unit, eye position did not drift beyond the recordable area for any of the participants. The recorded eye movement data were transformed to $x, y$-coordinate positions for time points sampled at a rate of $60 \mathrm{~Hz}$. Saccades were identified as eye movements with velocities between $30^{\circ}$ and $750^{\circ}$ of visual angle per second. A saccade onset was defined as the time point when an eye movement velocity was greater than $30^{\circ}$ of visual angle per second after $80 \mathrm{msec}$ following stimulus onset and the preceding eye movement velocity was less than $30^{\circ}$ of visual angle per second (i.e., a fixation). A saccade offset was defined as the time point when the next eye movement velocity was less than $30^{\circ}$ of visual angle per second following saccade onset.

Trials were excluded from analyses if the eyes' fixation location at target presentation was greater than $1.5^{\circ}$ of horizontal visual angle from the fixation cross, the first saccade following the abrupt onset of the target was either initiated in less than $80 \mathrm{msec}$ following target presentation, or the saccade did not exceed $5^{\circ}$ of horizontal visual angle from the center fixation. Of the acceptable trials, the first saccade following the target 
onset that was directed either toward or away from the asterisk, in response to a green or red cue, designated a trial as a correctly executed prosaccade or antisaccade, respectively. Erroneous prosaccades were trials that cued the participant to make an antisaccade (red eyes), but a prosaccade was made. Erroneous antisaccades were trials that cued the participant to make a prosaccade (green eyes), but an antisaccade was made.

\section{Magnetoencephalography}

Magnetic fields were recorded using a 151-channel whole-head neuromagnetometer system (VSM-Med Tech Inc.), with detection coils uniformly spaced by $31 \mathrm{~mm}$ distance on a helmet-shaped array. Sensors were configured as first-order axial gradiometers with $50 \mathrm{~mm}$ baseline. The spectral density of the intrinsic noise of each channel was about $5 \mathrm{fT} / \mathrm{Hz}$ in the frequency range above $1 \mathrm{~Hz}$. A participant's head position relative to the MEG sensor was monitored at the start and end of each recording. Head movements did not exceed $12 \mathrm{~mm}$ for any of the participants.

Magnetoencephalography single-trial data with large artifacts (greater than 1.5 pT) were removed. Based on the combined EMM and MEG criteria for acceptable inclusion, on average, 64\% (standard error $=6 \%$ ) of the trials were included for further analyses. Erroneous antisaccades were too few for MEG analyses to be conducted; thus, those trials were discarded. The MEG data were then epoched and averaged relative to the saccade onset time obtained from the EMM data (saccadelocked). The saccade-locked data were then modeled using equivalent current dipole (ECD) modeling and event-related synthetic aperture magnetometry (ERSAM) procedures (Cheyne, Bakhtazad, \& Gaetz, 2006; Hamalainen, Hari, Ilmoniemi, Knuutila, \& Lounasmaa, 1993). Saccade-locked data were modeled separately for prosaccades, antisaccades, and erroneous prosaccades. ECD modeling was used because it is a standard for localizing neural generators of MEG and EEG data. ER-SAM was used because it is based on nonlinear beamforming (Cheyne et al., 2006) that overcomes a limitation of ECD modeling by reducing correlations in the data in order to suppress interacting sources. This procedure can better localize multiple overlapping sources within the brain that are likely to occur in higher-order perceptual and cognitive processes. The two analyses provide converging evidence for the neural regions that support saccade execution and suppression.

For ECD modeling, two approaches were taken. For our first approach, we attempted to model the MEG field data by searching through the time course and fitting the peak of dipolar field patterns with one or two dipoles. We then fit the residual fields with subsequent dipoles. This approach led to large variability in dipole locations and signal-space projected waveforms among subjects and did not adequately explain the field data.
We therefore took a second approach, which accounted for the field data and were consistent across most of the subjects. A pair of dipoles (symmetrically constrained to a hemisphere) located in the orbits accounted for the field pattern over the frontal regions at saccade onset. Another pair located within the central-parietal region accounted for the residual field pattern at saccade onset (modeled variance $>85 \%$ ). A single dipole located in the occipital lobe accounted for the neural responses that occurred approximately 100 and 200 msec following saccade onset; hereafter referred to as the S1 and S2, respectively (modeled variance $>85 \%$ ). We did attempt to model a pair of symmetrically linked dipoles for the S1 and S2 field topographies; however, they consistently fused to a single midline location in the occipital cortex among subjects. We therefore used a single dipole to explain these topographies. Data from two participants could not be adequately modeled (i.e., less the $75 \%$ modeled variance) and were not included in subsequent analyses. This was likely due to low signal-to-noise ratios resulting from averaging a limited number $(<25)$ of trials that remained after artifact rejection and rejection of trials on the basis of the above eye movement criteria. For the remaining 9 of the 11 participants' data, frontal, central, and occipital dipoles were combined to form a single model that accounted for greater than $75 \%$ of the variance between -100 and 200 msec relative to saccade onset (see Figure 2). For these nine acceptable datasets, individual MEG saccade-locked field data were then projected down into the dipoles using the signal-space projection method (Uusitalo \& Ilmoniemi 1997). The dipole source waveforms were averaged across all participants and a 30-Hz low-pass filter was applied. To obtain significant level differences in source waveforms between saccade types, data were averaged in 25-msec intervals from -500 to $500 \mathrm{msec}$ relative to saccade onset. These data were then used to calculate null distributions for differences between saccade types by 1024 resamplings with replacement using the bootstrap method (Efron \& Tibshirani, 1993). Significance thresholds were calculated as two-tailed confidence intervals at $95 \%, 99 \%$, and $99.9 \%$ (i.e., alpha levels of .05, .01, and .001 ) of the null distributions for differences between a priori defined contrasts of prosaccades versus antisaccades, prosaccades versus erroneous prosaccades, and antisaccades versus erroneous prosaccades.

Event-related synthetic aperture magnetometry was used to localize the cortical activity observed at approximately $100 \mathrm{msec}$ prior to saccade onset (hereafter referred to as the presaccadic field or PSF) and S2 that showed the largest differential responses between prosaccades and antisaccades. To obtain the ER-SAM maps, sensor weighting factors were estimated on the basis of the single-state, pseudo- $Z$ SAM spatial filtering of the singletrial data (low-pass filtered at $30 \mathrm{~Hz}$ ) between -500 and 500 msec relative to prosaccade or antisaccade onset. These weighting factors were then applied to the 
averaged saccade-locked fields from 151 MEG sensors to obtain virtual channel waveforms at each $10 \times 10 \times$ $10 \mathrm{~mm}$ voxels throughout the whole brain. To avoid waveform deconstruction during averaging, the absolute pseudo $Z$-value of the PSF and S2 time points for each virtual channel was used to obtain a pseudo- $Z$ SAM map of each participant's brain activity. Each participant's SAM map was then transformed into common anatomical space, using structural MRI normalization transforms, calculated in SPM99, before averaging functional SAM maps across participants. Group-averaged SAM maps were calculated for prosaccades, antisaccades, erroneous prosaccades, and the comparisons between antisaccades and (correct and erroneous) prosaccades at $-100 \mathrm{msec}$ (PSF) and 200 msec (S2) relative to saccade onset.

\section{RESULTS}

\section{Eye Movements}

Antisaccade execution requires inhibition of a reflexive prosaccade (Olk \& Kingstone, 2003). This was evident as a decrease in accuracy (68\%) on antisaccade compared to prosaccade trials $(84 \% ; t=2.43, d f=10, p<.05)$ and an increase in saccadic latency for antisaccades (313 msec) compared to either correctly generated prosaccades (257 msec) or prosaccades that were erroneously generated in response to the antisaccade cue (erroneous prosaccades; 247 msec; $F=16.6, d f=2,20, p<.01$ ). Saccadic latencies were not different between prosaccades and erroneous prosaccades, demonstrating that erroneous prosaccades reflect ineffective suppression of the reflexive prosaccade motor output. Average saccade duration was $71 \mathrm{msec}$, and no significant differences were observed between saccades types. There were not enough erroneous antisaccades to conduct analyses on the MEG data; as such, the analyses below are exclusively for the prosaccades, antisaccades, and erroneous prosaccades.

\section{MEG Fields}

Inspection of the MEG field topographies revealed neural responses in the frontal sensors that were locked to saccade onset and likely reflect the horizontal translation of the eyes (e.g., eye movement fields, or EMF, as shown in a representative subject in Figure 1). Preparatory activity for all saccades was reflected as a ramping PSF in the central sensor waveforms, as shown in Figure 1 (McDowell et al., 2005; Clementz et al., 2001; Everling \& Fischer, 1998; Evdokimidis, Liakopoulos, Constantinidis, \& Papageorgiou, 1996). Following saccade onset, two distinct neural responses were evident for each saccade type from the occipital sensors at approximately 100 and $200 \mathrm{msec}$ postsaccade; these are labeled as S1 and S2, respectively, in Figure 1.

\section{Dipole Source Activity}

Neural activation at saccade onset and the S2 was localized to brain regions using dipole modeling and signalspace projection procedures (Uusitalo \& Ilmoniemi 1997); further dipole modeling at the S1 latency did not substantially improve the source model. Figure 2A shows a representative participant's source model with dipoles located within the frontal cortices (Dipoles 1 and 2), the visual cortex (Dipole 3), and the eyes (Dipoles 4 and 5); Figure 2B shows the group-averaged source waveforms localized within the orbits as well as the frontal and visual cortices that precede and follow saccade onset. Descriptions of the presaccadic and postsaccadic neural responses are outlined in turn, below.

\section{Presaccadic Responses}

Prior to saccade onset, source waveforms from frontal and visual regions show evidence for suppression of a
Figure 1. A representative participant's MEG field topography at 88 msec after prosaccade onset is shown on the left. MEG sensor waveforms averaged across prosaccade, antisaccade, or erroneous prosaccade trials, with respect to saccade onset, are shown on the right. $\mathrm{EMF}=$ eye movement field recorded over prefrontal/ orbital regions; PSF = presaccadic field recorded over frontal-central regions; $\mathrm{S} 1$ = first peak of postsaccadic field recorded over occipital regions; S2 = second peak of postsaccadic field recorded over occipital regions.

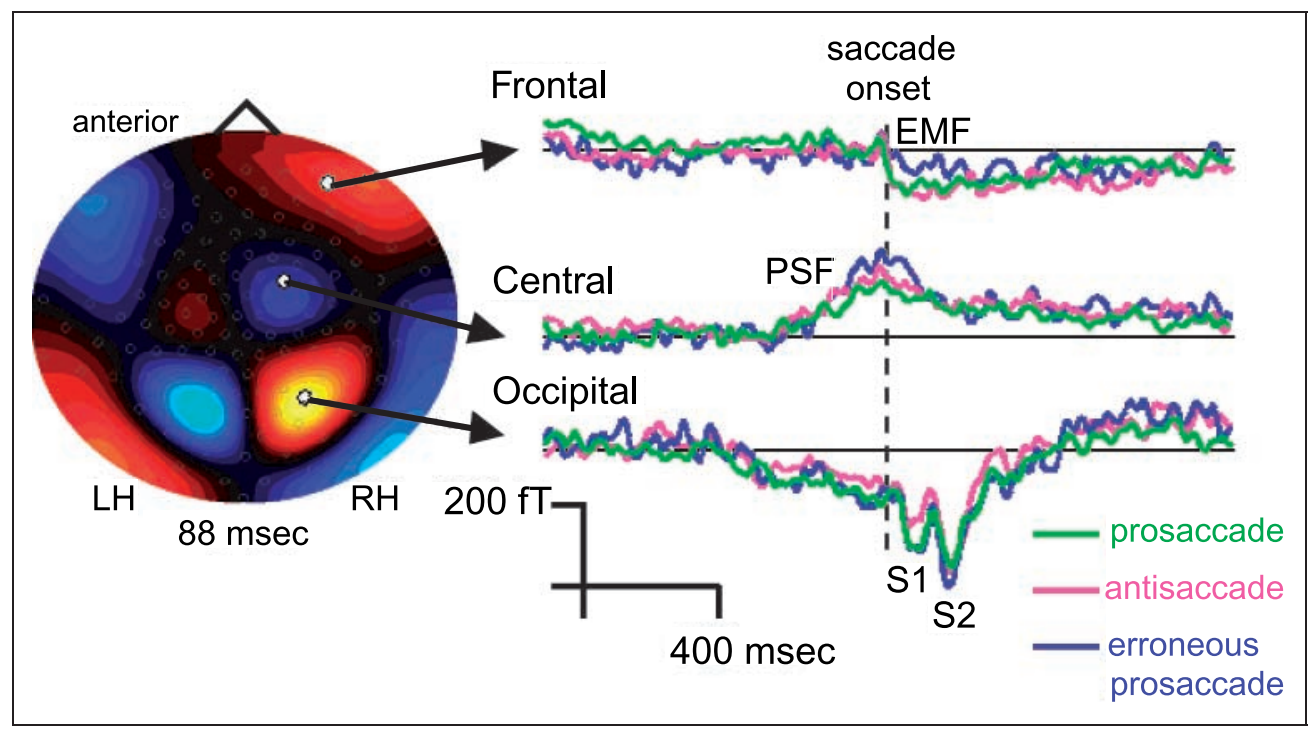




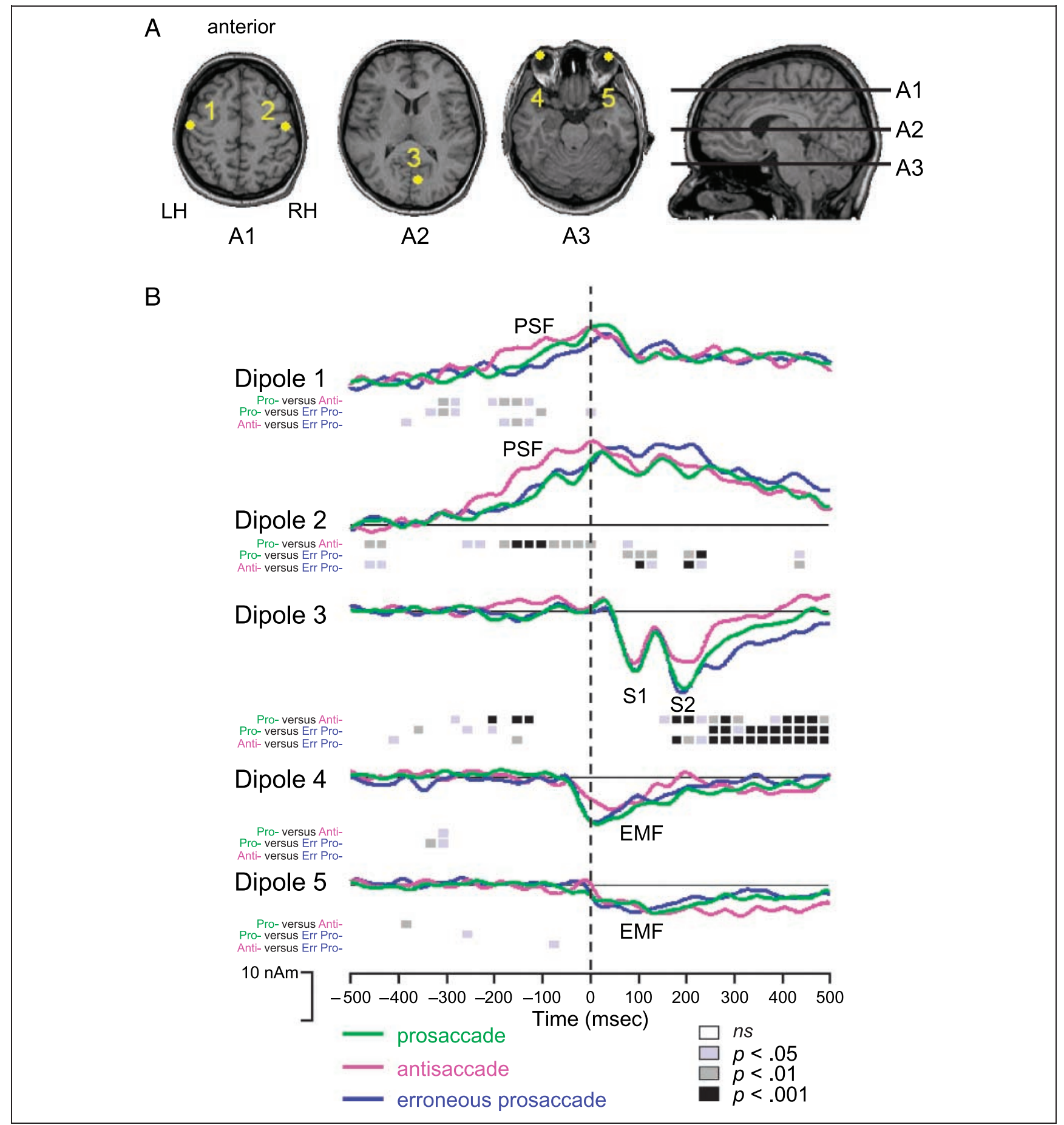

Figure 2. Saccade-locked source activity for prosaccades, antisaccades, and erroneous prosaccades based on equivalent current dipole (ECD) modeling. (A) Source model of dipoles (yellow dots) located in frontal cortices (1 and 2), the visual cortex (3), and the eyes (4 and 5). (B) Group-averaged saccade-locked source waveforms for Dipoles 1-5. Significance levels (based on null distributions calculated using the bootstrap sampling method by Efron \& Tibshirani, 1993) are given for a priori pairwise contrasts averaged across 25 -msec intervals between -500 and 500 msec with respect to saccade onset.

reflexive motor plan that would otherwise direct the eyes toward a visual target (Dipoles 1, 2, and 5; Figure 2B) (McDowell et al., 2005; Thickbroom, Knezevic, Carroll, \& Mastaglia, 1991). Responses were significantly and selectively greater prior to saccade onset for correctly executed antisaccades compared to prosaccades or erroneous prosaccades. In particular, activity in the frontal cortices distinguished an error from a correctly generated antisaccade as early as $200 \mathrm{msec}$ prior to saccade onset. No differences were observed in the waveforms of the frontal or visual dipoles between prosaccades and erroneous prosaccades, suggesting that 
the erroneous prosaccades result from ineffective suppression of the reflexive motor plan. Alternatively, correctly generated antisaccades differ from the other two eye movements in that the spatial locations of two goal locations must be successfully processed and reconciled in order for the correct saccadic eye movement to occur-the location of the target and the location opposite that of the target-whereas only one spatial location would need to be processed and maintained in order to generate a (erroneous) prosaccade. Therefore, the frontal and visual activity $200 \mathrm{msec}$ prior to saccade onset may reflect the processing of multiple spatial locations to which the eyes may be directed.

\section{Postsaccadic Responses}

At saccade onset, responses localized to the orbits did not distinguish among the saccade types (prosaccade, antisaccade, erroneous prosaccade). Such activity merely reflects the magnetic fields generated by the translation of the eyes rather than underlying neuronal responses.

Postsaccadic responses from frontal cortices indicated the generation of an error-related feedback signal that may serve to initiate corrective eye movements. Postsaccadic frontal responses for erroneous prosaccades were significantly larger compared to correctly executed prosaccades or antisaccades between 60 and $250 \mathrm{msec}$, whereas prosaccades and antisaccades did not differ (Figure 2B).

The initial postsaccadic visual response at the $\mathrm{S} 1$ (see Figure 2B, Dipole 3) did not distinguish among the saccade types. However, at and following the S2, visual responses differentiated the goal-directed (antisaccades) from the reflexive saccades (prosaccades and erroneous prosaccades) (Figure 2B). Following the S2, a third peak was observed around $270 \mathrm{msec}$ that was significantly different for the erroneous prosaccades compared to either of the correctly executed saccade responses (prosaccades, antisaccades). It would appear then that the neural responses from frontal sources that differentiate correct from incorrect saccades preceded similar responses from the visual cortex.

\section{ER-SAM Source Activity}

The above localizations of neural activity from dipole modeling were compared to the localization findings from ER-SAM (Cheyne et al., 2006). In particular, ER-SAM was used to describe the neural regions underlying the PSF and S2 responses that differentiated the saccade types (prosaccades, antisaccades, and erroneous prosaccades).

The PSF was localized to FEFs using ER-SAM, which is consistent with the dipole locations in the frontal cortices. ER-SAM revealed greater cortical activity within the right FEF for antisaccades compared to prosaccades at $100 \mathrm{msec}$ prior to saccade onset (precentral gyrus; Talairach \& Tournoux, 1988, coordinates $=39-649 \mathrm{~mm}$ ). Greater activity was observed within the right middle frontal gyrus (Talairach \& Tournoux, 1988, coordinates $=311452 \mathrm{~mm}$ ) for antisaccades compared to erroneous prosaccades at 100 msec prior to saccade onset (see Figure 3A).

The neural substrates underlying the $\mathrm{S} 2$ responses were localized to areas that have been implicated in visual processing and in the remapping of target location in space from retinal coordinate information (Law et al., 1998). Activity for prosaccades, antisaccades, and erroneous prosaccades were localized to the precuneus (Talairach \& Tournoux, 1988, coordinates $=31-65$ $41 \mathrm{~mm})$, cuneus $(-5-8216 \mathrm{~mm})$, left and right middle occipital gyri $(-33-728 \mathrm{~mm},-21-84-3 \mathrm{~mm})$, right fusiform gyrus (39-72-19 $\mathrm{mm}$ ), and right posterior superior temporal gyrus ( $58-326 \mathrm{~mm}$; see Figure $3 \mathrm{~B})$. Prosaccades and erroneous prosaccades had greater activity in the precuneus, cuneus, and right middle temporal gyrus at $200 \mathrm{msec}$ after saccade onset.

\section{DISCUSSION}

Simultaneous EMM-MEG recordings revealed the spatiotemporal brain dynamics underlying saccade suppression and error-related feedback. Recording saccades with an eyetracker allowed us to use only those trials in which acceptable saccades occurred, that is, those trials in which saccades of a particular velocity were generated in response to, and not prior to presentation of, the cue, and were directed near the target location. EMM recordings permitted MEG trial differentiation based on whether correct or incorrect eye movements were made, thereby allowing examination of the neural underpinnings of subsequent successful versus unsuccessful inhibition of reflexive saccades and error-related feedback signals.

Presaccadic FEF activity was observed for each saccade type (prosaccade, antisaccade, erroneous prosaccade) and distinguished subsequent reflexive eye movements from correctly generated antisaccades, suggesting a role for this region in the successful inhibition of erroneous saccades (Olk, Chang, Kingstone, \& Ro, 2006; Machado \& Rafal, 2004). The FEF activity for the difference between antisaccades and (erroneous) prosaccades was slightly more anterior, similar to what has been observed in tasks that require the inhibition of a reflexive response, whether the response is made via pointing or saccades (Connolly et al., 2000). Connolly et al. (2000) suggest that such an anterior shift may reflect the covert orienting of attention and the processing of spatial information regarding the goal location. Presaccadic responses from the visual cortex were also related to subsequent successful inhibition of reflexive eye movements (Ioannides et al., 2004; Connolly et al., 2002). These findings are consistent with those from electrophysiological recordings in primates (Bruce \& Goldberg, 1985), as well as neuroimaging studies in humans (McDowell et al., 2005; Zhang \& Barash, 2004). 
Figure 3. Group-averaged event-related SAM maps of cortical source activities to prosaccades, antisaccades, and erroneous prosaccades, as well as contrasts of antisaccades versus either prosaccades or erroneous prosaccades. (A) Cortical activity at 100 msec prior to saccade onset (i.e., PSF). (B) Cortical activity $200 \mathrm{msec}$ after saccade onset (i.e., S2). Pseudo $Z$-values represent the ratio of signal-to-noise power of the evoked response.

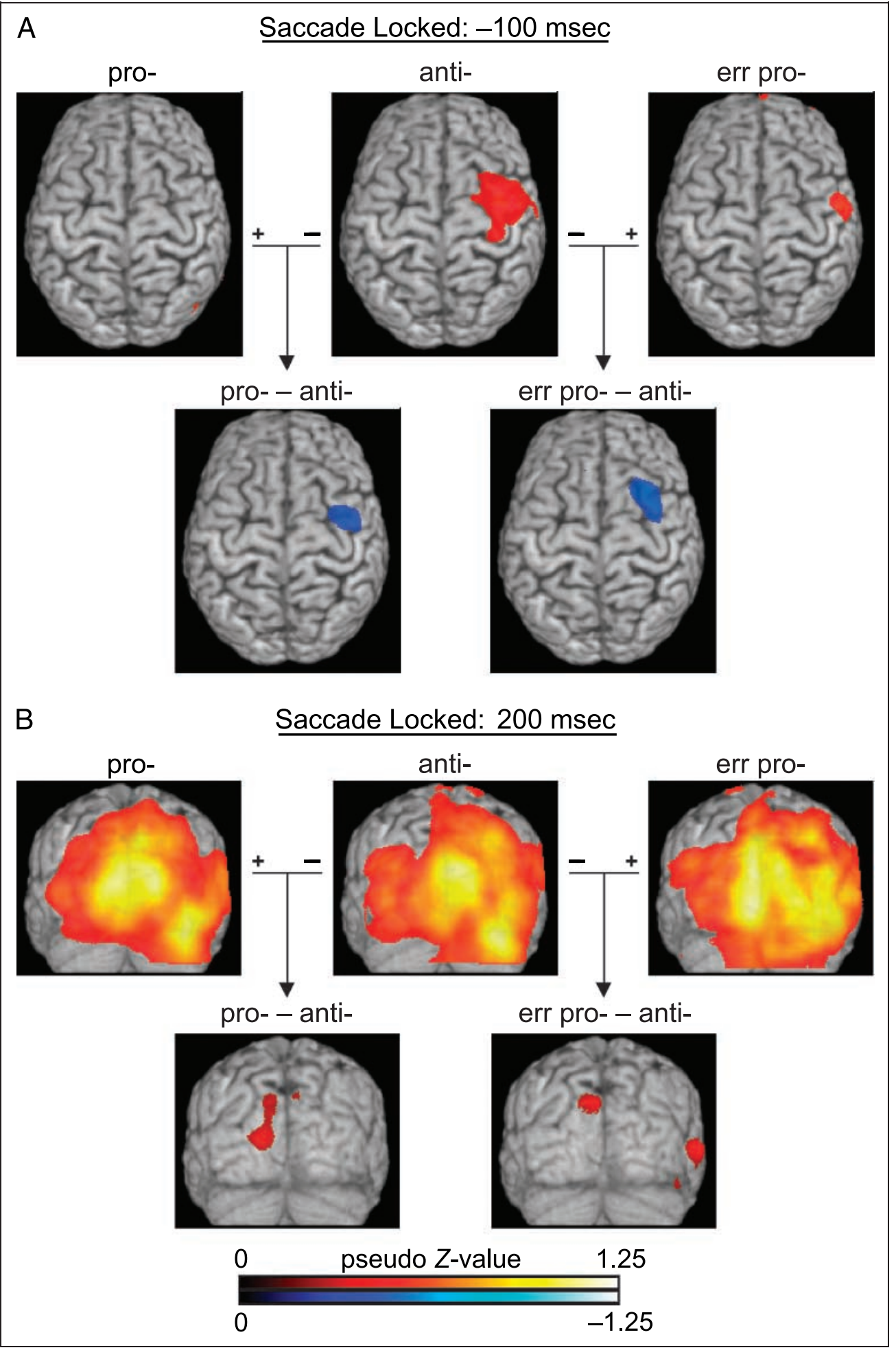

A Saccade Locked: $-100 \mathrm{msec}$

B

Regarding the relative temporal sequence of neural activation, activity from the FEF and visual cortices appeared to come on-line simultaneously, approximately $200 \mathrm{msec}$ prior to the saccade execution. These responses may be indicative of neural coupling of the eye movement signal with visual-spatial information regarding the intended goal location in order to produce a goal-directed saccade with the appropriate ve- locity and distance. The difference in presaccadic responses from the FEF and visual cortices for antisaccades versus erroneous and correct prosaccades may reflect the processing of one goal location in the case of prosaccades (that of the target location) and the processing of two goal locations in the case of correctly generated antisaccades (that of the target location and the location opposite to that of the target). Erroneous 
prosaccades may occur due to the processing and maintenance of only the location of the target and not the location opposite to that of the target, resulting in an incorrect eye movement.

In addition to examining the relative timing of neural responses that occur prior to saccade onset, the current study expanded upon previous research by examining the neural responses that come on-line in humans following the generation of correct and incorrect saccades.

A neural response stemming from regions within the visual cortex was evident at $100 \mathrm{msec}$ following saccade onset (S1). This neural activation did not distinguish the saccade types, and thus, may be indicative of saccadic suppression; as the eyes move across the display, the blurring of the visual field is not perceived, rather, the viewer perceives a stable environment. There is some indication that saccadic suppression may be governed via responses from the visual cortex (Sylvester, Haynes, \& Rees, 2005; Thickbroom et al., 1991). An additional neural response was evident at $200 \mathrm{msec}$ following saccade onset that distinguished goal-directed (antisaccades) from reflexive (prosaccades, erroneous prosaccades) saccades. This S2 response was localized to the cuneus, precuneus, and middle temporal gyrus. Due to the role of these regions in mapping eye position and integrating such information with target spatial locations (Deutschlander et al., 2005; Makino, Yokosawa, Takeda, \& Kumada, 2004; Vidnyanszky, Gulyas, \& Roland, 2000), this neural response difference may reflect differences in comparison of the current eye position with an endogenously generated goal location. However, if the S2 response reflects processing of the current spatial location with either the endogenously versus exogenously generated target location, it is not clear why the S2 responses for the prosaccades (exogenous goal location) and erroneous prosaccades (exogenous and endogenous goal locations) do not differ. It may be that the endogenous goal location was not maintained by regions within the visual cortex in those instances in which erroneous prosaccades occur, thereby resulting in only a comparison between the current position and the exogenous goal location, similar to prosaccades. Note that it is also just prior to the S2 response in which the FEF appears to generate an error-related signal, as outlined below. This signal may be the re-production of the endogenous goal location that is then propagated through feedback signals to regions within the visual cortex and is revealed as a sustained neural response following the S2 for the erroneous prosaccades.

Examination of postsaccadic responses further permitted investigation of the role of the FEF and posterior regions in error-related feedback. Postsaccadic responses from the FEF and visual cortices each differentiated correct from erroneous eye movement responses. These findings are consistent with those from Bruce and Goldberg (1985), which showed sustained FEF activity in primates that occurred following an error. The novel finding from the current work stems from the observation that the error-related activity from the FEF preceded that from visual regions. The relative timing of the observed responses in the FEF and visual cortices suggests that the FEF governs a feedback signal that projects back to, and combines with, signals from the posterior cortices, which provide target coordinate information in order to drive a corrective saccadic response. Future work that examines the neural responses locked to secondary corrective saccades rather than initial saccades would further outline the spatio-temporal dynamics related to corrective eye movements. To appropriately determine whether the postsaccadic frontal responses are indicative of an error-related signal only or are indicative of an errorrelated signal plus a signal that initiates a corrective response, one would need to compare those trials in which a corrective eye movement response was made to those trials in which a corrective saccade was not made. To further subdivide the erroneous prosaccades in the current analyses into those two groups was not possible here due to the low number of trials. Therefore, the suggestion here is that these frontal responses are indicative of an error-related response, and perhaps, also drive a corrective saccade, but this latter speculation would need to be borne out in further studies. In addition, analyses that are time-locked to the corrective saccadic response may also reveal activity in regions such as the anterior cingulate cortex, which are typically observed in response to error monitoring and correction (Debener et al., 2005; Nieuwenhuis, Ridderinkhof, Blom, Band, \& Kok, 2001; Gehring \& Knight, 2000; Badgaiyan \& Posner, 1998).

Our findings from ER-SAM appear to reveal laterality effects in activated neural regions presaccade and postsaccade onset. However, it is not clear whether these laterality effects would be observed regardless of saccade direction; the analyses here included unequal numbers of leftward and rightward saccades, and there were too few trials to analyze the effects of saccade direction on the current findings across all of the conditions.

The findings of presaccadic and postsaccadic FEF activity that distinguish antisaccades from prosaccades are consistent with other findings from neuroimaging (McDowell et al., 2005; Munoz \& Everling, 2004; Everling \& Fischer, 1998; Doricchi et al., 1997; Everling et al., 1997; Schlag-Rey, Amador, Sanchez, \& Schlag, 1997; Sweeney et al., 1996; O’Driscoll et al., 1995). However, single-unit recordings from Everling and Munoz (2000) revealed that FEF saccade neurons actually decrease in firing rate prior to the execution of antisaccades compared to prosaccades. The authors suggest that the neuronal responses observed in neuroimaging studies may reflect an increase in activation of inhibitory interneurons that act upon neurons that generate the saccadic response. Therefore, the presaccadic responses within the FEF, as observed here, may reflect neuronal inhibition that prevents a reflexive response from occurring. However, the increase in FEF activity following 
saccade execution may reflect activation in saccaderelated neurons that initiate a corrective eye movement rather than reflecting continued activation of inhibitory interneurons. Our findings show an increase in postsaccadic FEF activity for the erroneous prosaccades compared to the correctly generated pro- and antisaccades, suggesting that this response is related to feedback rather than to continued inhibition of saccade neurons. This would lead to the prediction that although similar MEG responses in the FEFs are observed pre- and postsaccadic onset, these responses have different underlying causes, and thus, single-unit recordings would reveal a decrease in FEF saccade neuron activity in the presaccade period for goal-directed versus reflexive saccades, but an increase in activity of the FEF saccade neurons for erroneous compared to correct saccades in the postsaccade period. Indeed, there is already some indication for sustained FEF activity following erroneous prosaccades from Bruce and Goldberg (1985). Moreover, it would be expected that the postsaccadic increase in FEF activity would be related to the generation of a corrective saccade.

It may be the case that the observed pre- and postsaccadic neural responses that differentiated reflexive from goal-directed, and correct from incorrect, saccade responses are confounded by differences in saccade latency. Certainly, confounds due to differences in saccade latency would be an issue had the data been locked to the onset of the prosaccade or antisaccade stimulus cue. If particular neural responses are tied to saccadic onset, analyzing neural activity with respect to the onset of a stimulus cue would have caused saccade-specific responses to become smoothed and possibly averaged out in the data analysis. This may be particularly true for findings from fMRI and PET studies in which the temporal resolution is insufficient to observe distinct responses that occur on the order of milliseconds and that are precisely time-locked to a saccade. For this reason, we chose to use MEG to investigate the underlying neural activity supporting saccadic generation and suppression; using this technique, neural activity could be analyzed with respect to saccade onset. Confounds due to saccade latency cannot be definitively ruled out in the present work, however, it is reasonable to presume that the added processing requirements needed to inhibit a reflexive response in order to correctly generate an antisaccade are reflected in increased activation within the FEFs prior to saccade onset and in a subsequent increase in saccade latency (Olk \& Kingstone, 2003). In this case, it is not an increase in saccade latency per se that causes increased neural responses within the FEF, but rather, the increase in neural responses in the FEF and the increase in saccade latency have a common underlying cause - the additional processing requirements needed to inhibit a reflexive glance.

Moreover, differences in saccadic latency are unlikely to result in the observed postsaccadic differences. Neu- ral activation in response to prosaccade, antisaccades, and erroneous prosaccades were similar immediately following saccade onset (i.e., S1), which might not be expected if confounds due to saccade latency continue to exert residual effects following saccade onset. It is only later, at the S2, that differences between the saccade types emerge.

It should be noted that we did not find significant activation originating from the DLPFC that distinguished goal-directed from reflexive saccades, whereas such activation was observed in the MEG study of McDowell et al. (2005). However, other neuroimaging studies have not reported significant DLPFC activation in antisaccade and memory-guided saccade tasks (e.g., Sugiura et al., 2004; Kimmig et al., 2001; Law et al., 1998). In addition, the lack of significant activation in the DLPFC here may not be surprising when the findings from DeSouza, Menon, and Everling (2003) are considered. DeSouza et al. found that DLPFC activation distinguishing prosaccades from antisaccades was only apparent when data were analyzed with reference to the onset of the cue stimulus, which instructed viewers as to whether they were to make a prosaccade or antisaccade in response to the target onset. If the DLPFC is uniquely involved in a preparatory set, rather than in the generation of saccades, our current analyses that locked neural activity to saccade onset, rather than to instructional cue onset, may have obscured any responses from the DLPFC. As well, it is likely that activation from the DLPFC in response to the instructional cue in the current work may have resolved by the time the target onset is presented. This would not likely have been the case for the McDowell et al. study, which used blocked rather than random presentations of prosaccade and antisaccade trials, thus instructional cues that designated whether viewers were to make a prosaccade or antisaccade were not embedded within the trial. In the McDowell et al. study, viewers were given a fixation cross followed by the target presentation. As a result, signals from the DLPFC that reflect preparatory set may temporally overlap signals that govern the eye movement itself because there is not enough time for the preparatory signals to be resolved. Furthermore, the use of a gap condition in the current work, in contrast to the McDowell et al. study, may contribute to the resolution of preparatory signals.

One additional difference between the current work and the work of DeSouza et al. (2003) concerns the activation observed within the FEFs. FEF activation was also apparent only during the instructional period in the DeSouza et al. study, whereas we found significant responses from the FEF immediately prior to saccade onset. The authors acknowledged that the lack of FEF activity distinguishing prosaccades from antisaccades in their study was surprising, and suggested that the temporal resolution of fMRI may not have been sensitive to detect FEF activity. The increased temporal precision 
of MEG in the current work may have allowed the differential FEF activity to be observed.

\section{Concluding Remarks}

Eye movement monitoring has been used for decades as a tool for investigating issues surrounding attention (Godijn \& Theeuwes, 2003; Irwin, Colcombe, Kramer, \& Hahn, 2000), language processing and production (Starr \& Rayner, 2001; Griffin \& Bock, 2000), scene perception (Loftus \& Mackworth, 1978), and memory (Ryan \& Cohen, 2004; Ryan, Althoff, Whitlow, \& Cohen, 2000), among others. MEG has been used to answer questions about brain dynamics of cognitive and perceptual processes, including those involved in literacy (Herdman et al., 2006; Simos et al., 2002), attention (Ishii et al., 1999), face processing (Halgren, Raij, Marinkovic, Jousmaki, \& Hari, 2000; Watanabe, Kakigi, Koyama, \& Kirino, 1999), working memory (Tesche \& Karhu, 2000), and long-term spatial memory (de Araujo, Baffa, \& Wakai, 2002). When used in conjunction here, EMM-MEG recordings allowed us to lock neural activity to behavior with temporal precision, thereby revealing the location and sequence of brain activity that support such higherorder cognitive processes. Specifically, the current work outlined the spatio-temporal dynamics underlying the suppression of reflexive eye movement behavior and the generation of error-related feedback signals. This work provides the first empirical demonstration of remote eyetracking within an MEG environment. Finally, this work provides an example of a within-paradigm converging evidence approach that can be used to answer questions regarding the sequence of dynamic brain networks that are used to support particular classes of behavior, thereby providing a new method to assess cognition in a variety of fields.

\section{Acknowledgments}

We thank Endel Tulving, Terry Picton, and Sandra Moses for their assistance. This work was supported by a grant from the Natural Sciences and Engineering Research Council of Canada (J. D. R.)

Reprint requests should be sent to Dr. Jennifer D. Ryan, Scientist, The Rotman Research Institute, Baycrest, 3560 Bathurst Street, Toronto, ON, Canada M6A 2E1, or via e-mail: jryan@ rotman-baycrest.on.ca.

\section{REFERENCES}

Badgaiyan, R. D., \& Posner, M. I. (1998). Mapping the cingulate cortex in response selection and monitoring. Neuroimage, 7, 255-260.

Bialystok, E., Craik, F. I. M., \& Ryan, J. D. (2006). Executive control in a modified anti-saccade task: Effects of aging and bilingualism. Journal of Experimental Psychology: Learning, Memory, and Cognition, 32, 1341-1354.

Bruce, C. J., \& Goldberg, M. E. (1985). Primate frontal eye fields: I. Single neurons discharging before saccades. Journal of Neurophysiology, 53, 603-635.

Cheyne, D., Bakhtazad, L., \& Gaetz, W. (2006). Spatiotemporal mapping of cortical activity accompanying voluntary movements using an event-related beamforming approach. Human Brain Mapping, 27, 213-229.

Clementz, B. A., McDowell, J. E., \& Stewart, S. E. (2001). Timing and magnitude of frontal activity differentiates refixation and anti-saccade performance. NeuroReport, 12, 1863-1868.

Connolly, J. D., Goodale, M. A., Desouza, J. F., Menon, R. S., \& Vilis, T. (2000). A comparison of frontoparietal fMRI activation during anti-saccades and anti-pointing. Journal of Neurophysiology, 84, 1645-1655.

Connolly, J. D., Goodale, M. A., Menon, R. S., \& Munoz, D. P. (2002). Human fMRI evidence for the neural correlates of preparatory set. Nature Neuroscience, 5, 1345-1352.

Cornelissen, F. W., Kimmig, H., Schira, M., Rutschmann, R. M., Maguire, R. P., Broerse, A., et al. (2002). Event-related fMRI responses in the human frontal eye fields in a randomized pro- and antisaccade task. Experimental Brain Research, 145, 270-274.

de Araujo, D. B., Baffa, O., \& Wakai, R. T. (2002).

Theta oscillations and human navigation: A magnetoencephalography study. Journal of Cognitive Neuroscience, 14, 70-78.

Debener, S., Ullsperger, M., Siegel, M., Fiehler, K., von Cramon, D. Y., \& Engel, A. K. (2005). Trial-by-trial coupling of concurrent electroencephalogram and functional magnetic resonance imaging identifies the dynamics of performance monitoring. Journal of Neuroscience, 25, 11730-11737.

DeSouza, J. F., Menon, R. S., \& Everling, S. (2003). Preparatory set associated with pro-saccades and anti-saccades in humans investigated with event-related FMRI. Journal of Neurophysiology, 89, 1016-1023.

Deutschlander, A., Marx, E., Stephan, T., Riedel, E., Wiesmann, M., Dieterich, M., et al. (2005). Asymmetric modulation of human visual cortex activity during 10 degrees lateral gaze (fMRI study). Neuroimage, 28, 4-13.

Doricchi, F., Perani, D., Incoccia, C., Grassi, F., Cappa, S. F., Bettinardi, V., et al. (1997). Neural control of fast-regular saccades and antisaccades: An investigation using positron emission tomography. Experimental Brain Research, 116, 50-62.

Dorris, M. C., \& Munoz, D. P. (1995). A neural correlate for the gap effect on saccadic reaction times in monkey. Journal of Neurophysiology, 73, 2558-2562.

Efron, B., \& Tibshirani, R. J. (1993). An introduction to the bootstrap. New York: Chapman \& Hall.

Evdokimidis, I., Liakopoulos, D., Constantinidis, T. S., \& Papageorgiou, C. (1996). Cortical potentials with antisaccades. Electroencephalography and Clinical Neurophysiology, 98, 377-384.

Everling, S., \& Fischer, B. (1998). The antisaccade: A review of basic research and clinical studies. Neuropsychologia, 36, 885-899.

Everling, S., Krappmann, P., \& Flohr, H. (1997). Cortical potentials preceding pro- and antisaccades in man. Electroencephalography and Clinical Neurophysiology, 102, 356-362.

Everling, S., \& Munoz, D. P. (2000). Neuronal correlates for preparatory set associated with pro-saccades and anti-saccades in the primate frontal eye field. Journal of Neuroscience, 20, 387-400.

Forbes, K., \& Klein, R. M. (1996). The magnitude of the fixation offset effect with endogenously and exogenously controlled saccades. Journal of Cognitive Neuroscience, 8, 344-352. 
Ford, K. A., Goltz, H. C., Brown, M. R., \& Everling, S. (2005). Neural processes associated with antisaccade task performance investigated with event-related fMRI. Journal of Neurophysiology, 94, 429-440.

Gehring, W. J., \& Knight, R. T. (2000). Prefrontal-cingulate interactions in action monitoring. Nature Neuroscience, 3, 516-520.

Godijn, R., \& Theeuwes, J. (2003). Parallel allocation of attention prior to the execution of saccade sequences. Journal of Experimental Psychology: Human Perception and Performance, 29, 882-896.

Griffin, Z. M., \& Bock, K. (2000). What the eyes say about speaking. Psychological Science, 11, 274-279.

Guitton, D., Buchtel, H. A., \& Douglas, R. M. (1985). Frontal lobe lesions in man cause difficulties in suppressing reflexive glances and in generating goal-directed saccades. Experimental Brain Research, 58, 455-472.

Halgren, E., Raij, T., Marinkovic, K., Jousmaki, V., \& Hari, R. (2000). Cognitive response profile of the human fusiform face area as determined by MEG. Cerebral Cortex, 10, 69-81.

Hamalainen, M., Hari, R., Ilmoniemi, R. J., Knuutila, J., \& Lounasmaa, O. V. (1993). MagnetoencephalographyTheory, instrumentation, and applications to noninvasive studies of the working human brain. Review, Modern Physician, 65, 413-496.

Herdman, A. T., Fujioka, T., Chau, W., Ross, B., Pantev, C., \& Picton, T. W. (2006). Cortical oscillations related to processing congruent and incongruent grapheme-phoneme pairs. Neuroscience Letters, 399, 61-66.

Hikosaka, O., \& Wurtz, R. H. (1985). Modification of saccadic eye movements by GABA-related substances: I. Effect of muscimol and bicuculline in monkey superior colliculus. Journal of Neurophysiology, 53, 266-291.

Ioannides, A. A., Corsi-Cabrera, M., Fenwick, P. B., del Rio Portilla, Y., Laskaris, N. A., Khurshudyan, A., et al. (2004). MEG tomography of human cortex and brainstem activity in waking and REM sleep saccades. Cerebral Cortex, 14, 56-72.

Irwin, D. E., Colcombe, A. M., Kramer, A. F., \& Hahn, S. (2000). Attentional and oculomotor capture by onset, luminance and color singletons. Vision Research, 40, $1443-1458$

Ishii, R., Shinosaki, K., Ukai, S., Inouye, T., Ishihara, T., Yoshimine, T., et al. (1999). Medial prefrontal cortex generates frontal midline theta rhythm. NeuroReport, 10, 675-679.

Kimmig, H., Greenlee, M. W., Huethe, F., \& Mergner, T. (1999). MR-eyetracker: A new method for eye movement recording in functional magnetic resonance imaging. Experimental Brain Research, 126, 443-449.

Kurtzberg, D., \& Vaughan, H. G., Jr. (1982). Topographic analysis of human cortical potentials preceding self-initiated and visually triggered saccades. Brain Research, 243, 1-9.

Law, I., Svarer, C., Rostrup, E., \& Paulson, O. B. (1998). Parieto-occipital cortex activation during self-generated eye movements in the dark. Brain, 121, 2189-2200.

Lee, C., Rohrer, W. H., \& Sparks, D. L. (1988). Population coding of saccadic eye movements by neurons in the superior colliculus. Nature, 332, 357-360.

Loftus, G. R., \& Mackworth, N. H. (1978). Cognitive determinants of fixation location during picture viewing. Journal of Experimental Psychology: Human Perception and Performance, 4, 565-572.

Machado, L., \& Rafal, R. D. (2004). Control of fixation and saccades during an anti-saccade task: An investigation in humans with chronic lesions of the oculomotor cortex. Experimental Brain Research, 156, 55-63.
Makino, Y., Yokosawa, K., Takeda, Y., \& Kumada, T. (2004). Visual search and memory search engage extensive overlapping cerebral cortices: An fMRI study. Neuroimage, 23, 525-533.

Matsuda, T., Matsuura, M., Ohkubo, T., Ohkubo, H., Matsushima, E., Inoue, K., et al. (2004). Functional MRI mapping of brain activation during visually guided saccades and antisaccades: Cortical and subcortical networks. Psychiatry Research, 131, 147-155.

Mays, L. E., \& Sparks, D. L. (1980). Dissociation of visual and saccade-related responses in superior colliculus neurons. Journal of Neurophysiology, 43, 207-232.

McDowell, J. E., Kissler, J. M., Berg, P., Dyckman, K. A., Gao, Y., Rockstroh, B., et al. (2005). Electroencephalography/ magnetoencephalography study of cortical activities preceding prosaccades and antisaccades. NeuroReport, 16, 663-668.

Munoz, D. P., Broughton, J. R., Goldring, J. E., \& Armstrong, I. T. (1998). Age-related performance of human subjects on saccadic eye movement tasks. Experimental Brain Research, 121, 391-400.

Munoz, D. P., \& Everling, S. (2004). Look away: The anti-saccade task and the voluntary control of eye movement. Nature Reviews: Neuroscience, 5, 218-228.

Nieuwenhuis, S., Ridderinkhof, K. R., Blom, J., Band, G. P., \& Kok, A. (2001). Error-related brain potentials are differentially related to awareness of response errors: Evidence from an antisaccade task. Psychophysiology, 38, 752-760.

O'Driscoll, G. A., Alpert, N. M., Matthysse, S. W., Levy, D. L., Rauch, S. L., \& Holzman, P. S. (1995). Functional neuroanatomy of antisaccade eye movements investigated with positron emission tomography. Proceedings of the National Academy of Sciences, U.S.A., 92, 925-929.

Olk, B., Chang, E., Kingstone, A., \& Ro, T. (2006). Modulation of antisaccades by transcranial magnetic stimulation of the human frontal eye field. Cerebral Cortex, 16, 76-82.

Olk, B., \& Kingstone, A. (2003). Why are antisaccades slower than prosaccades? A novel finding using a new paradigm. NeuroReport, 14, 151-155.

Pierrot-Deseilligny, C., Rivaud, S., Gaymard, B., \& Agid, Y. (1991). Cortical control of reflexive visually-guided saccades Brain, 114, 1473-1485.

Ryan, J. D., Althoff, R. R., Whitlow, S., \& Cohen, N. J. (2000). Amnesia is a deficit in relational memory. Psychological Science, 11, 454-461.

Ryan, J. D., \& Cohen, N. J. (2004). The nature of change detection and online representations of scenes. Journal of Experimental Psychology: Human Perception and Performance, 30, 988-1015.

Schiller, P. H., \& Stryker, M. (1972). Single-unit recording and stimulation in superior colliculus of the alert rhesus monkey. Journal of Neurophysiology, 35, 915-924.

Schiller, P. H., Stryker, M., Cynader, M., \& Berman, N. (1974). Response characteristics of single cells in the monkey superior colliculus following ablation or cooling of visual cortex. Journal of Neurophysiology, 37, 181-194.

Schiller, P. H., True, S. D., \& Conway, J. L. (1980). Deficits in eye movements following frontal eye-field and superior colliculus ablations. Journal of Neurophysiology, 44, 1175-1189.

Schlag-Rey, M., Amador, N., Sanchez, H., \& Schlag, J. (1997). Antisaccade performance predicted by neuronal activity in the supplementary eye field. Nature, 390, 398-401.

Simos, P. G., Breier, J. I., Fletcher, J. M., Foorman, B. R., Castillo, E. M., \& Papanicolaou, A. C. (2002). Brain mechanisms for reading words and pseudowords: An integrated approach. Cerebral Cortex, 12, 297-305. 
Starr, M. S., \& Rayner, K. (2001). Eye movements during reading: Some current controversies. Trends in Cognitive Sciences, 5, 156-163.

Sugiura, M., Watanabe, J., Maeda, Y., Matsue, Y., Fukuda, H., \& Kawashimi, R. (2004). Different roles of the frontal and parietal regions in memory-guided saccade: A PCA approach on time course of BOLD changes. Human Brain Mapping, 23, 129-139.

Sweeney, J. A., Mintun, M. A., Kwee, S., Wiseman, M. B., Brown, D. L., Rosenberg, D. R., et al. (1996). Positron emission tomography study of voluntary saccadic eye movements and spatial working memory. Journal of Neurophysiology, 75, 454-468.

Sylvester, R., Haynes, J. D., \& Rees, G. (2005). Saccades differentially modulate human LGN and V1 responses in the presence and absence of visual stimulation. Current Biology, 15, 37-41.

Talairach, J., \& Tournoux, P. (1988). Co-planar stereotaxic atlas of the human brain. Stuttgart, Germany: Verlag.

Tesche, C. D., \& Karhu, J. (2000). Theta oscillations index human hippocampal activation during a working memory task. Proceedings of the National Academy of Sciences, U.S.A., 97, 919-924.

Thickbroom, G. W., Knezevic, W., Carroll, W. M., \& Mastaglia, F. L. (1991). Saccade onset and offset lambda waves: Relation to pattern movement visually evoked potentials. Brain Research, 551, 150-156.

Uusitalo, M. A., \& Ilmoniemi, R. J. (1997). Signal-space projection method for separating MEG or EEG into components. Medical \& Biological Engineering $\mathcal{E}$ Computing, 35, 135-140.

Vidnyanszky, Z., Gulyas, B., \& Roland, P. E. (2000). Visual exploration of form and position with identical stimuli: Functional anatomy with PET. Human Brain Mapping, 11, 104-116.

Watanabe, S., Kakigi, R., Koyama, S., \& Kirino, E. (1999). Human face perception traced by magneto- and electro-encephalography. Brain Research, Cognitive Brain Research, 8, 125-142.

Zhang, M., \& Barash, S. (2004). Persistent LIP activity in memory antisaccades: Working memory for a sensorimotor transformation. Journal of Neurophysiology, 91, 1424-1441. 